\title{
Laboratory Animal
}

National Cancer Institute

\section{Source}

National Cancer Institute. Laboratory Animal. NCI Thesaurus. Code C14183.

A grouping of those animals that are commonly used as subjects in laboratory research. 